\title{
Trajectories of the Single Moving Equivalent Dipole in Subjects With Left Fascicular Block
}

\author{
Vito Starc ${ }^{1}$, Cees A Swenne ${ }^{2}$ \\ ${ }^{1}$ University of Ljubljana, Faculty of Medicine, Ljubljana, Slovenia \\ ${ }^{2}$ Cardiology Department, Leiden University Medical Center, Leiden, The Netherlands
}

\begin{abstract}
We explored a potential clinical use of our application based on a spherically bounded model with homogeneous volume conductor to determine the location, orientation and strength of a single moving equivalent dipole (SMED) in 12-lead ECGs. We hypothesized that it would affect the location of the SMEDs in conduction defects.

Using ECG criteria of AHA/ACCF/HRS, we studied subjects with left anterior fascicular block (LAFB, $n=10)$ and those with left posterior fascicular block (LPFB, $n=17)$, contrasting them with subjects with normal ECGs $(n=42)$. The SMEDs were assessed in 5-min supine resting 12-lead ECGs with a time resolution of $1 \mathrm{~ms}$.

The heart axis through the centers of the derived atrial and ventricular SMED locations showed similar spatial orientation as that obtained by MRI studies. In LAFB and $\angle P F B$ we found that the direction of the SMED trajectory and the SMED orientation rotate in the opposite sense and with different patterns, suggesting that these patterns could help in further discrimination of both blocks.

This initial study with SMED assessment shows promising so far unexplored ECG-derived information. Further research in ECGs with various pathology is needed to investigate its possible diagnostic applicability.
\end{abstract}

\section{Introduction}

At CinC 2017 we presented an application based on a spherically bounded model with homogeneous volume conductor to determine location, strength, and orientation of a single moving equivalent dipole (SMED) in 12-lead ECGs [1]. The model estimates (inverse solution) SMEDs from potentials at the lead locations on the spherical surface adapted to a template torso. To initially explore its potential clinical use, we wanted to verify that the SMEDs in conduction defects will be in other locations than those in a healthy heart. To this purpose, we studied ECGs with left fascicular blocks after relating the SMED orientation to the heart.

\section{Subjects and methods}

Using the model we studied subjects in sinus rhythm that satisfied ECG criteria for the left anterior fascicular block (LAFB, $n=15$ ) and those for the left posterior fascicular block (LPFB, $n=17$ ), and contrasted these with apparently healthy individuals with normal ECGs, excluding athletes $(n=271)$. According to AHA/ACCF/ HRS recommendation [2], the criteria for LAFB included frontal plane axis between $-45^{\circ}$ and $-90^{\circ}$, $\mathrm{qR}$ pattern in lead aVL, R-peak time in lead aVL of $45 \mathrm{~ms}$ or more, and QRS duration less than $120 \mathrm{~ms}$. Those criteria for LPFB considered frontal plane axis between $90^{\circ}$ and $180^{\circ}$ in adults, $\mathrm{rS}$ pattern in leads I and aVL, qR pattern in leads III and aVF, and QRS duration less than $120 \mathrm{~ms}$. All subjects were selected from our database used previously [3], containing an unselected group of 1050 subjects.

\subsection{Determination of the SMEDs}

In our model representing the electrical activity of the heart derived from the general solution of Laplace's equation, surface potentials are proportional to the dot product of the dipole and the lead vectors of the 12-lead ECG. The model estimates (inverse solution) the dipole and its location by minimizing the objective function that represents the error between the calculated model potentials and the measured potentials. Potentials are obtained at the electrode locations of the standard 12-lead ECG on the spherical surface with a radius of $15 \mathrm{~cm}$, adapted to a template torso.

The SMEDs were determined in 5-min supine resting 12-lead ECGs with a time resolution of $1 \mathrm{~ms}$ (Cardiax/ Cardiosoft). The $\mathrm{T}$ wave was subtracted from the $\mathrm{P}$ wave before the QRS onset ( $T$ wave cancellation) to eliminate superposition of the $\mathrm{T}$ wave on the $\mathrm{P}$ wave. For each ECG sample, nine potentials (VR, VL, VF, V1..V6) evaluated concerning Wilson's central terminal were used to determine the corresponding SMED (dipole strength, orientation, and its location). 


\subsection{A model of the heart for the study of the SMED dynamics}

To study the position and orientation of SMEDs concerning the ventricular walls, a heart model with its 3D orientation was necessary to build. The left ventricle (LV) was represented as a rotational ellipsoid with the short axis $(b=3.5 \mathrm{~cm})$ rotated around its long axis $(a=5 \mathrm{~cm})$ and truncated at the atrioventricular $(\mathrm{AV})$ plane $1.5 \mathrm{~cm}$ above the ellipsoid origin. The right ventricle was represented similarly, using only one half of the longitudinally cut ellipsoid attached to the LV. We assumed that the long axis is oriented similarly to that one based on data from Odille $\mathrm{F}$ et al. [5]. The short axis was assumed to lie in the axial plane, symmetrically regarding the model heart.

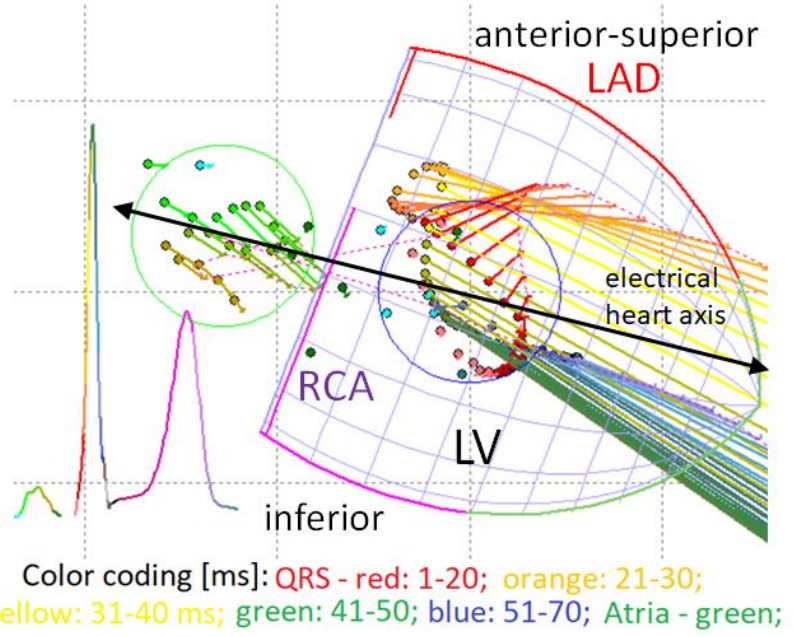

Figure 1. The heart electrical long axis (black line) and the referential model heart in the RAO view. The heart axis connects the centers of the atrial and ventricular SMED locations (surrounded by green and red circles, respectively). The SMED strength signal on the left has the same color coding as dipoles (the $\mathrm{T}$ wave is not shown). Dipoles are presented by arrows (orientation) and closed circles (location), respectively. LAD, left anterior descending artery; RCA, right coronary artery;

For evaluation of the SMED orientation concerning the heart walls, the spatial orientation of the heart and proper locations of the SMEDs inside the heart were necessary. We assumed that the SMED trajectory of the first $60 \mathrm{~ms}$ of QRS is centered at the point a half way from the origin of the ellipsoidal LV to the septum, and speculated that the center of SMEDs of atrial activation lies on the long axis of LV. By connecting both SMED centers, we estimated the spatial orientation of the heart electrical axis [Fig. 1], expressed by its elevation and azimuth. After comparing our orientation angles with those from the MRI study [3], these angles were further corrected for bias between the studies and used as referential.

\subsection{SMED trajectory and SMED rotation}

The SMED trajectories were constructed from a set of SMED locations providing the direction of movement, which was related to the instantaneous SMED orientation. It was evaluated by utilizing the planar angles $\xi$ and $\psi$, of the direction of movement and the SMED orientation, respectively, obtained by projecting them onto a plane. Specifically, we used the atrioventricular (AV) plane (the LAO view), and the septal plane (the RAO view), with orientations given by the electrical heart axis. Both planar angles were referenced relative to the short axis position.

The SMED trajectory exhibited a curvilinear path with the SMED orientation (a planar angle $\psi$ ) in all directions concerning the direction of movement (an angle $\xi$ ). Several typical patterns emerged during the QRS. In the $\psi$ flip pattern, a nearly linear trajectory path experienced a sudden change in planar angle $\psi$ of up to $180^{\circ}$ within 5 ms [Fig 2A]. The SMED trajectory often exhibited a trajectory loop (TL) with an angle $\xi$ rotating either in the clockwise ( $\xi \downarrow$ ) [Fig. 2B] or in the counter-clockwise ( $\xi \uparrow)$ sense [Fig. 2C]. Rotations in angle $\xi$ were accompanied by rotations in angle $\psi$ (the SMED orientation) either in the clockwise $(\psi \downarrow)$ or the counter-clockwise $(\psi \uparrow)$ sense.

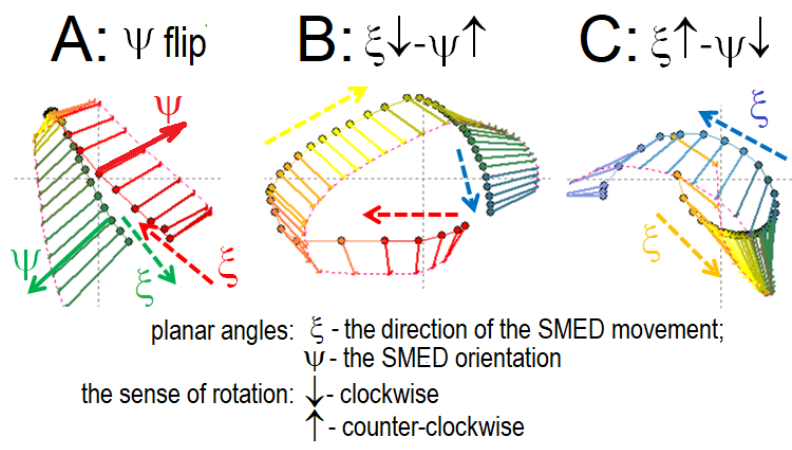

Figure 2. The dynamics of the SMED trajectory and the SMED orientation. The SMED orientation is shown by solid arrows (angle $\psi$ ), and the direction of movement (angle $\xi$ ) by broken colored arrows. A: The $\psi$ flip pattern with a linear trajectory path in the AV plane. B, C: combined $\xi \downarrow-\psi \uparrow$ and $\xi \uparrow-\psi \downarrow$ patterns, respectively, both in the septal plane. Dipolar strength in log values.

To quantify changes $\psi$ in our subjects, we determined the points $P_{1}$ and $P_{2}$ in the AV plane [Fig .3]. $P_{1}$ was defined at the time instant $t_{1}$ after the QRS onset when the SMED strength exceeded $10 \%$ of the maximal value reached during QRS, and $\psi$ attained the $\psi_{1}$. Though in many cases $\xi$ exhibited a zig-zag pattern, $\psi$ did not change considerably during the initial phase until the appearance of a $\psi$ flip, which occurred at time $t_{2}$. Ten ms later $\left(\mathrm{t}_{2}+10 \mathrm{~ms}\right)$ at the point $\mathrm{P}_{2}, \psi$ was stabilized at the value of $\psi_{2}$. We also determined the distance $d_{12}$ between the points $\mathrm{P}_{1}$ and $\mathrm{P}_{2}$ and the angle $\xi_{12}$ from $\mathrm{P}_{1}$ and $\mathrm{P}_{2}$. 


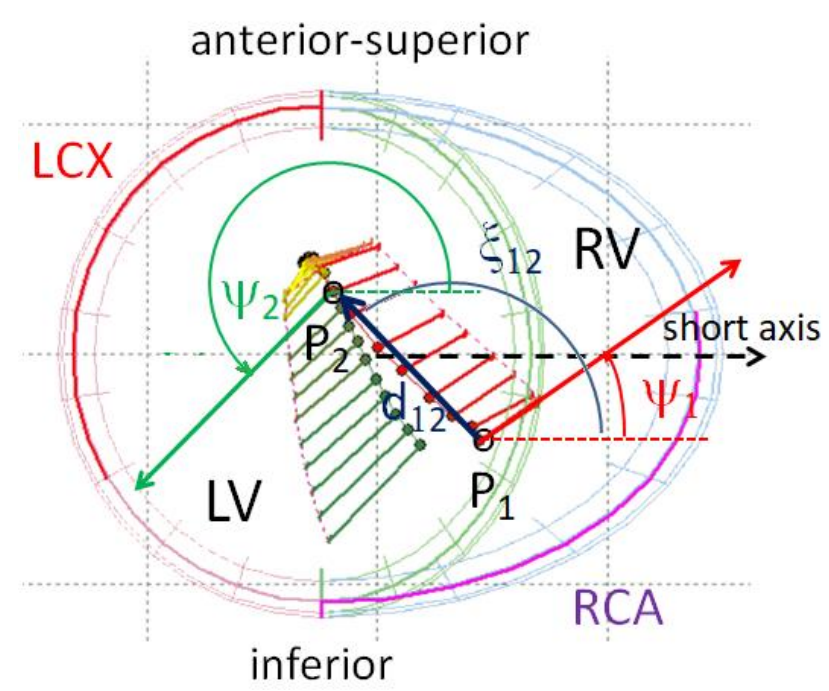

Figure 3. The model of LV in the AV plane (LAO view) with SMEDs of the first $70 \mathrm{~ms}$ of QRS. $\psi_{1}$ and $\psi_{2}$, orientation angles of the SMEDs located at $\mathrm{P}_{1}$ and $\mathrm{P}_{2} ; \xi_{12}$ and $d_{12}$, direction angle and distance from $P_{1}$ to $P_{2}$; LCX, the left circumflex artery. See also Fig. 1.

\section{Results}

The determination of the electrical heart long axis in space using the mean SMED locations of the $\mathrm{P}$ wave and QRS provided the value for elevation in the control group of $-6.8^{\circ}$ and those for azimuth of around $-54.7^{\circ}$ (Table 1 ), which are biased from the referential values of $-21.3^{\circ}$ and $-57.1^{\circ}$ by nearly $14.5^{\circ}$ and $2.4^{\circ}$, respectively, but with similar variances. There were no statistically significant differences between the LAFB and LPFB groups vs. control except that for elevation between LAFB and Ctrl.

Table 1. SMED dynamics in the AV plane

\begin{tabular}{|c|c|c|c|c|c|c|}
\hline Variab & & ${ }^{0} \mathrm{Ctrl}$ & ${ }^{1} L A F B$ & ${ }^{2} L P F B$ & $p_{01}$ & $p_{02}$ \\
\hline $\mathrm{N}$ & & 271 & 15 & 18 & & \\
\hline \multirow[t]{2}{*}{ elevation } & Mean & -6.8 & -7.1 & -11.6 & 0.007 & 0.50 \\
\hline & $\pm \mathrm{SD}$ & 14.0 & 10.7 & 20.4 & $*$ & NS \\
\hline \multirow[t]{2}{*}{ azimuth } & Mean & -54.7 & -55.3 & -51.2 & 0.84 & 0.99 \\
\hline & $\pm \mathrm{SD}$ & 14.7 & 24.5 & 12.1 & NS & NS \\
\hline
\end{tabular}

$p_{01}$ and $p_{02}: p$ values of the statistical significance for differences between the control and LAFB groups, and between the control and $L P F B$ group, respectively

The SMED trajectory exhibited a curvilinear path with SMEDs oriented in all directions concerning the direction of movement, mostly at a larger angle, e.g., in the transverse direction and rarely along or at a small angle to the direction to the movement.

In the control group ( $\mathrm{Ctrl}, \mathrm{N}=271)$ in the projection onto the AV plane, the SMEDs at $\mathrm{P}_{1}$ were directed mainly toward the septum and slightly anteriorly at the mean angle $\psi_{1}$ of $21.5^{\circ}$ and later at $\mathrm{P}_{2}$ at the angle $\psi_{2}$ of $200^{\circ}$, when the trajectory covered the distance $\mathrm{d}_{12}$ of $1.7 \mathrm{~cm}$ in the direction $\xi_{12}$ of $189^{\circ}$ from $\mathrm{P}_{1}$ to $\mathrm{P}_{2}$.

In the septal plane, TL rotation of the SMEDs existed in $46(52 \%)$ cases all exhibiting $\xi \uparrow$, whereas in $33(37 \%)$ cases the loop was either degenerated into two antiparallel lines or a $\psi$ flip occurred (Table 2). The rotation of the SMED orientation showed $\psi \uparrow \uparrow$ pattern in 68 (76\%) cases, and the $\psi$ flip occurred in $11(12 \%)$ cases. In the rest of the cases, the rotation was indeterminate. The most frequent combination of the rotation sense of the TL and ED orientation was $\xi \uparrow-\psi \downarrow$, appearing in 41 (46\%) cases.

Table 2. Dynamics of the direction of the SMED trajectory and the SMED orientation in the septal plane

\begin{tabular}{cccc}
\hline Pattern & ${ }^{0}$ Ctrl & ${ }^{1}$ LAFB & ${ }^{2}$ LPFB \\
\hline $\mathrm{N}$ & 89 & 15 & 18 \\
$\xi \uparrow$ & $52 \%$ & 0 & $94 \%$ \\
$\xi \downarrow$ & & $87 \%$ & 0 \\
$\psi \uparrow$ & & $73 \%$ & 0 \\
$\psi \downarrow$ & $76 \%$ & 0 & $94 \%$ \\
$\xi \uparrow-\psi \downarrow$ & $46 \%$ & 0 & $89 \%$ \\
$\xi \downarrow-\psi \uparrow$ & 0 & $73 \%$ & 0 \\
\hline
\end{tabular}

$\xi$, trajectory direction angle; $\psi$, SMED orientation angle; $\uparrow$, counter-clockwise; $\downarrow$, clockwise

In the LAFB group $(\mathrm{N}=15)$ the projection of the SMEDs orientation onto the AV plane showed that SMEDs were initially oriented statistically significantly more toward the inferior LV wall than in Ctrl $\left(\psi_{1}:-58^{\circ}\right.$ vs. $21.5^{\circ}, \mathrm{p}<10^{-10}$ ), and those of the early middle phase more toward the anterosuperior wall than in Ctrl $\left(\psi_{2}\right.$ : $164^{\circ}$ vs. $200^{\circ}, \mathrm{p}=10^{-3}$ ). Also, the SMEDs moved from $\mathrm{P}_{1}$ to $\mathrm{P}_{2}$ with a smaller $\xi$ than in $\operatorname{Ctrl}\left(\xi_{12}: 150^{\circ}\right.$ vs. $189^{\circ}$, $\mathrm{p}=0.02)$ to a distance $\mathrm{d}_{12}=1.6 \mathrm{~cm}$ from $\mathrm{P}_{1}(1.7 \mathrm{~cm}$ in Ctrl, NS). In the septal plane (the RAO view), the dynamics of the SMED trajectories showed the following predominant patterns: $\xi \downarrow$ for TL rotation (13 cases, 87\%), $\psi \uparrow$ for SMED orientation (11 cases, 73\%), and $\xi \downarrow-\psi \uparrow$ (Fig. 2B, Table 2) for a combination (13 cases, $87 \%$ ).

In the LPFB group $(\mathrm{N}=18)$, the SMEDs $\mathrm{P}_{1}$ projected onto the $\mathrm{AV}$ plane were oriented more toward the anterior-superior $\mathrm{LV}$ wall than in $\mathrm{Ctrl}\left(\psi_{1}=+58^{\circ}\right.$ vs. $21.5^{\circ}, \mathrm{p}<10^{-10}$ ), and those at $\mathrm{P}_{2}$ pointed toward the inferior wall $\left(\psi_{2}=201\right.$ vs. $200^{\circ}$, NS). The SMEDs moved from $\mathrm{P}_{1}$ to $\mathrm{P}_{2}$ in the direction of $\xi_{12}=246^{\circ}\left(189^{\circ}\right.$ in Ctrl, $\mathrm{p}$ $=0.03)$, covering a distance $d_{12}=2.1 \mathrm{~cm}$ from $P_{1}(1.7 \mathrm{~cm}$ in Ctrl, $\mathrm{p}=0.02$ ). In the septal plane, the predominant patterns were: $\xi \uparrow$ for TL rotation (17 cases, 94\%), $\psi \downarrow$ for the SMED orientation (17 cases, 94\%), and $\xi \uparrow-\psi \downarrow$ (Fig. 2C) for a combination (16 cases, $89 \%$ ). 


\section{Discussion and conclusion}

This initial study with SMED assessment provided a distribution of SMEDs that is consistent with the anatomical orientation of the heart. It introduced the concept of the SMED trajectory often forming a loop, of which rotation sense, in combination with that of the SMED orientation, may help in discriminating the patterns of excitation in the heart.

Specifically, the method provided acceptable $\mathrm{P}$ wave SMED locations to get reasonable values for the heart orientation. The electrical heart axis obtained by our method is closely related to the spatial orientation of the anatomical axis of LV obtained by MRI [4], as suggested by a small bias between two methods of around $14.5^{\circ}$ for the elevation angle and around $2.4^{\circ}$ for the azimuth, and by comparable variances obtained from our study, and those of the angles $\theta_{\text {AX }}^{1}$ and $\theta_{\text {CORO [4]. The deviation }}^{1}$ from the referential angles might have arisen due to anatomical misalignment of the center of the SMED trajectory in atria from the LV long axis (Fig. 1) or is due to different populations studied. This speculation could be tested using MRI. Better agreement for the azimuth could be explained by more electrodes in the transverse plane.

The orientation of a SMED concerning a selected segment of the LV wall is more important than simply its orientation in 3-D space because it offers a ground for better physiological interpretation. It requires that the derived SMED locations need to be at the right places within the heart. The determination of SMEDs might be affected by many approximations used in the model, such as is a thorax in the form a spherical homogeneous conductor or inaccurate positions of the measuring electrodes or the uncertainty of a subject-specific orientation of the heart. We only speculate that a mild distortion of space by those approximations would minimally affect mutual relative positions of SMEDs.

Our study showed that in LAFB SMEDs are oriented initially toward the inferior LV wall and after 30 to $40 \mathrm{~ms}$ nearly in the opposite direction. Next, during QRS the SMED orientation rotates in the counter-clockwise sense, whereas in LPFB the behavior of SMEDs it represents the exact mirror picture of LAFB, initially pointing to the anterior-superior LV wall and later nearly to the opposite direction, with a clockwise rotation of the SMED orientation. These findings that are all concordant with the vectorcardiographic view [5] support the validity of our method. Also, with the finding that the SMED trajectory direction and the SMED orientation rotate in the opposite sense and with different patterns in LAFB and LPFB, our method could participate in extending of discrimination criteria in both blocks from the controls.

The SMEDs orientations were mostly aligned in the transverse direction of the SMED movement (Fig. 2), not as expected from the behavior of the depolarization wavefront. As a similar observation was found in another study using moving dipoles [5], we suggest verifying this finding using a large scale biophysical model.

Despite apparently good results when separating individuals of the LAFB or LPFB group from the controls, a word of caution is necessary. Both groups with fascicular blocks are rather small, comprising around $1.5 \%$ of the initial population and with individuals selected retrospectively. Using stronger criteria for frontal axis deviation $\left(>110^{\circ}\right)$ than those of AHA/ACCF/HRS [2], Elizari et al. [6] reported that pure LPFB is extremely rare, and mostly associated with the right bundle branch block. Retrospectively we found that 11 of the 18 subjects were actively engaged in the sports, and only 3 of them satisfied the stronger ECG criteria for LPFB. When considering the finding that $46 \%$ of the controls exhibited the same $\xi \uparrow-\psi \downarrow$ pattern, prevalent in the LPFB group, it seems that majority of our LPFB subjects were not true, but rather satisfied (mild) criteria for LPFB. We speculate that the SMED pattern could help in discrimination. For confirmation, a study with a larger LPFB group and stronger inclusion criteria should be performed.

By relating the SMED locations to the anatomy of the heart, this initial study with SMED assessment shows promising so far unexplored ECG-derived information. Further research in ECGs with various pathology is needed to investigate the possible applicability of this analysis in future ECG diagnostic algorithms.

\section{References}

[1] Starc V, Swenne CA. Spatial distribution and orientation of a single moving dipole computed in 12-lead ECGs in a healthy population using a spherically bounded model. Computing in Cardiology 2017; 44: pp.4.

[2] Surawicz B, Childers R, Deal BJ, Gettes LS, et al. AHA/ACCF/HRS recommendations for the standardization and interpretation of the electrocardiogram: part III. Circulation. 2009 Mar 17;119(10):e235-40..

[3] Starc V, Schlegel TT. Change in angular velocity at the end of the QRS loop aids the electrocardiographic detection of acute inferior myocardial infarction. Computing in Cardiology 2015; 42: 601-4.

[4] Odille F, Liu S, van Dam P, Felblinger J. Statistical variations of heart orientation in healthy adults. Computing Cardiol 2017; 44: pp. 4.

[5] Bystricky W. Identification of strict left bundle branch block using a moving dipole model. Computing in Cardiology 2018;45: pp 4 (in press).

[6] Elizari MV, Acunzo RS, Ferreiro M. Hemiblocks revisited. Circulation. 2007 Mar 6;115(9):1154-63.

Address for correspondence.

Vito Starc, MD, $\mathrm{PhD}$

Ljubljana University, Faculty of Medicine

Zaloska 4,

SI 1104 Ljubljana, Slovenia

E-mail: vito.starc@mf.uni-lj.si 